\title{
OPEN Gelsolin and dCryAB act downstream of muscle identity genes and contribute to preventing muscle splitting and branching in Drosophila
}

\author{
Benjamin Bertin, Yoan Renaud, Teresa Jagla, Guillaume Lavergne, Cristiana Dondi, \\ Jean-Philippe Da Ponte, Guillaume Junion ${ }^{\bowtie}$ Krzysztof Jagla ${ }^{\bowtie}$
}

A combinatorial code of identity transcription factors (iTFs) specifies the diversity of muscle types in Drosophila. We previously showed that two iTFs, Lms and Ap, play critical role in the identity of a subset of larval body wall muscles, the lateral transverse (LT) muscles. Intriguingly, a small portion of $a p$ and $I m s$ mutants displays an increased number of LT muscles, a phenotype that recalls pathological split muscle fibers in human. However, genes acting downstream of Ap and Lms to prevent these aberrant muscle feature are not known. Here, we applied a cell type specific translational profiling (TRAP) to identify gene expression signatures underlying identity of muscle subsets including the LT muscles. We found that Gelsolin (Gel) and $d C r y A B$, both encoding actin-interacting proteins, displayed LT muscle prevailing expression positively regulated by, the LT ITFs. Loss of $d C r y A B$ function resulted in LTs with irregular shape and occasional branched ends also observed in ap and lms mutant contexts. In contrast, enlarged and then split LTs with a greater number of myonuclei formed in Gel mutants while Gel gain of function resulted in unfused myoblasts, collectively indicating that Gel regulates LTs size and prevents splitting by limiting myoblast fusion. Thus, $d C r y A B$ and Gel act downstream of Lms and $A p$ and contribute to preventing $L T$ muscle branching and splitting. Our findings offer first clues to still unknown mechanisms of pathological muscle splitting commonly detected in human dystrophic muscles and causing muscle weakness.

Diversification of cell types is a fundamental process during the development of multicellular organisms and is essential in building functional organs. The muscle network in Drosophila embryos, composed of 30 muscle fibres per abdominal hemisegment, offers a tractable system for studying cell diversification. Despite common characteristics such as formation by myoblast fusion and the capacity to contract, each embryonic Drosophila muscle has a specific size, orientation, number of nuclei, attachment and innervation ${ }^{1}$. However, how these features are acquired at the muscle-specific level remains unclear, although it is generally accepted ${ }^{2,3}$ that the muscle founder cells (FCs), which are at the origin of muscle fibres, harbour all the information required for individual muscle identity. This information is thought to be provided by the combinatorial expression of identity genes encoding identity transcription factors (iTFs) ${ }^{4,5}$. They are all activated in subsets of muscle progenitors and/or FCs and share one important feature: their loss of function leads to the loss or aberrant properties of a muscle in which they are expressed ${ }^{1}$. Some identity genes such as lateral muscles scarcer $(l m s)^{6}$ show a restricted expression in lateral muscles only, whereas others, such as slouch (slou) display a broader expression pattern in ventral, lateral and dorsal domains ${ }^{7,8}$. Most Drosophila iTFs have their vertebrate counterparts, some of which (e.g. Org-1/TBX, Caup/IRX, Tup/ISL), play conserved roles during musculature specification in vertebrates ${ }^{9-11}$. Knowledge gained in Drosophila on iTFs and their downstream targets could thus cast light on how muscle diversification processes are regulated. It could also help to understand how aberrant muscle features such as branching and splitting are acquired. Whole genome approaches based on ChIP experiments have shown that iTFs directly regulate not only other identity genes, but also downstream regulators of muscle identity, the "realisator genes"12-14. We previously 
A

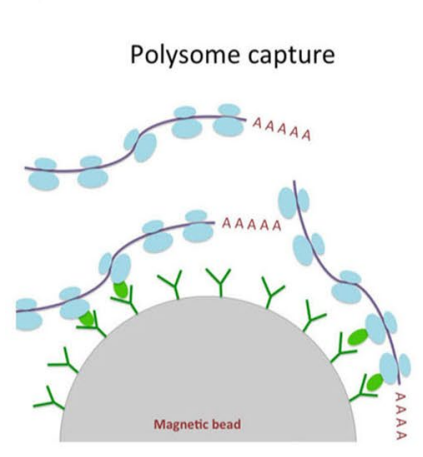

C

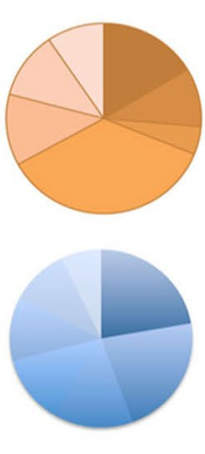

Im muscle structure development (69/285)

II muscle cell differentiation (42/126)

amyofibril assembly (20/45)

Emolecule metabolic process (151/961)

Esupramolecular fiber organization (51/259)

पmetabolism and energy (48/200)

$\square$ mitochondrial translation (40/95)

= biological regulation(801/4205)

= multicellular organism process (793/4021

= localization (519/2407)

= cell development (40/1824)

" nervous system development (412/1759)

= transport (375/1835)
D

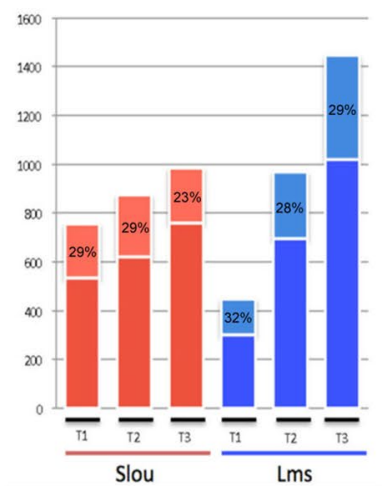

E $\frac{\text { Lms }}{\operatorname{Tr} 1 \quad \operatorname{Tr} 2} \frac{\text { Slou }}{\operatorname{Tr} 1 \quad T r 2}$

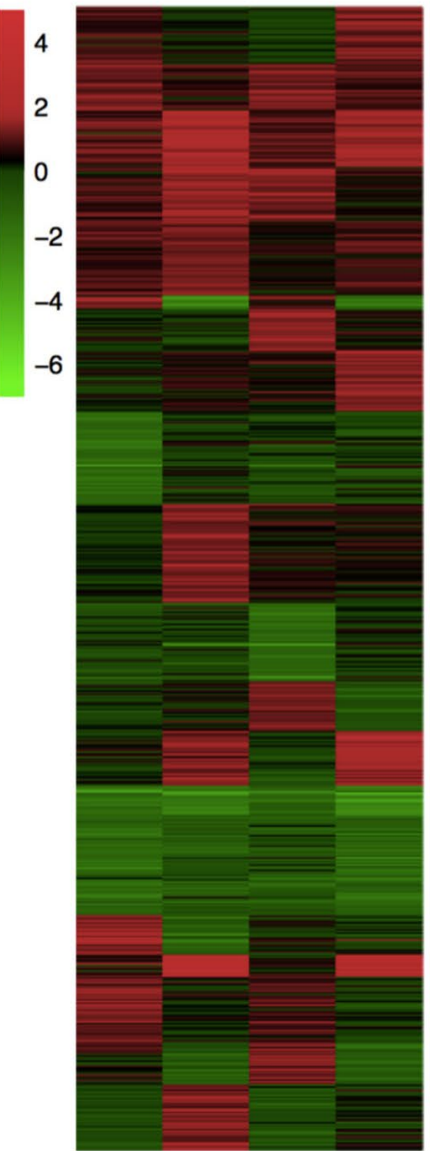

F GO - Molecular function
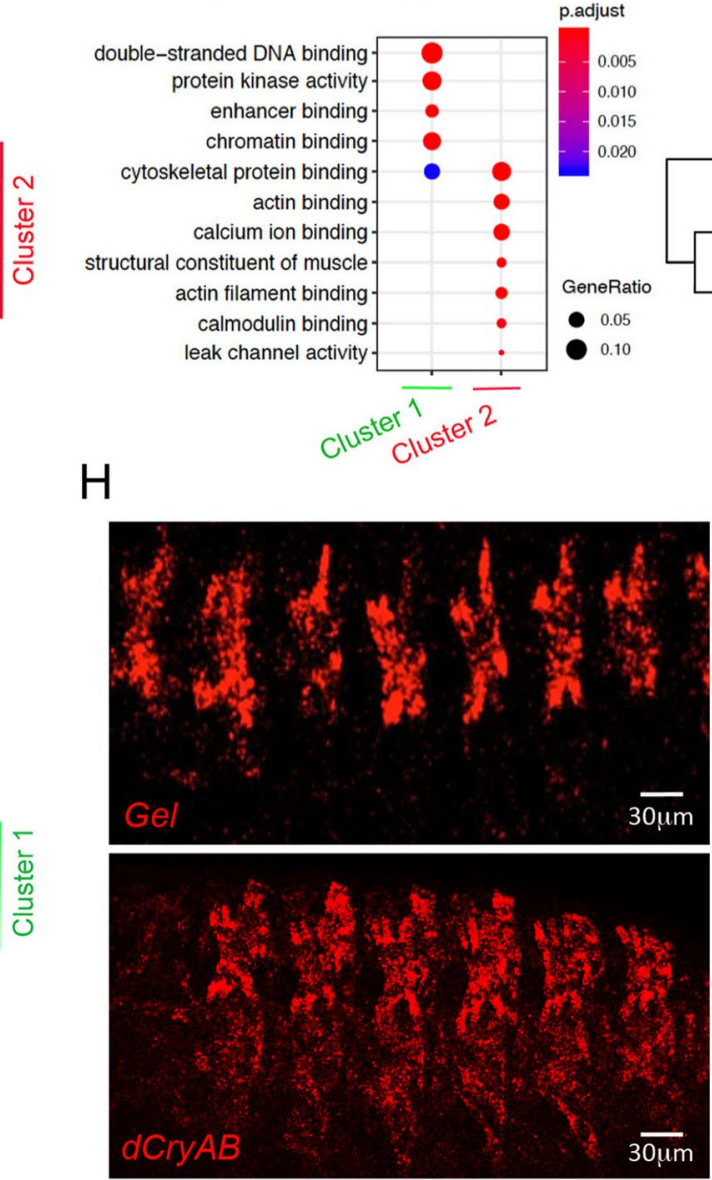

G

GO - Actin binding

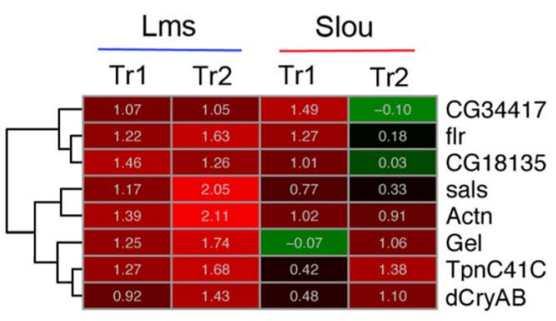

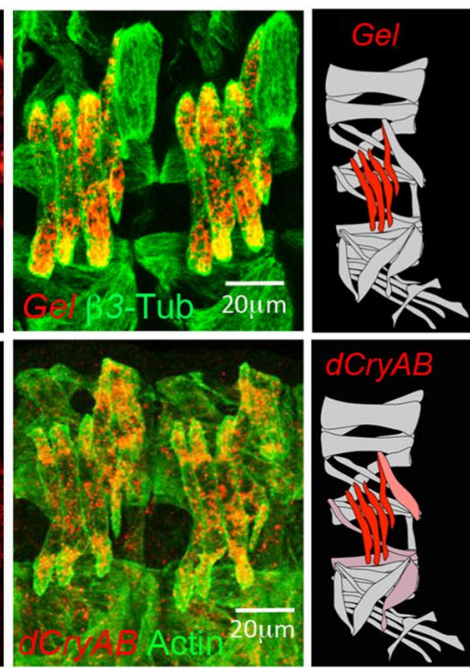


४Figure 1. Translational profiling of embryonic muscles using TRAP. (A) Schematic representation of cellspecific polysome capture during TRAP. (B) TRAP-targeted muscle populations revealed by anti-GFP immunostaining of stage 16 Slou $>$ RpL10a-eGFP and $L m s>R p L 10 a-e G F P$ embryos. (C) Pie chart showing distribution of GO terms (biological processes) for genes commonly up-regulated (orange) or down-regulated (blue) in Slou- and-Lms positive muscles and in all time windows. (D) Bar plots showing number of genes whose expression is commonly up $(\mathrm{FC}>2)$ in individual muscle subsets and in all muscles (dark red and dark blue) versus genes up-regulated exclusively in Slou (light red) and Lms (light blue) populations. Proportion of genes specific for each population at each time point are shown in light color boxes. (E) Heatmap showing the transition profiles of gene expression in Lms and Slou populations. Heatmap was generated using R package Pheatmap version 1.0.12 ; URL: https://cran.r-project.org/web/packages/pheatmap/index.html. Tr1 represents gene expression transition from the time window $\mathrm{T} 1$ to $\mathrm{T} 2$ and $\mathrm{Tr} 2$ the transition from $\mathrm{T} 2$ to $\mathrm{T} 3$. Cluster 1 (green) and cluster 2 (red) genes are indicated. (F) GO comparison of genes belonging to Cluster 1 and Cluster 2. Size of the circles represents the ratio of genes present in each GO category and color code the associated $\mathrm{p}$-value representing the probability of seeing at least $\mathrm{x}$ number of genes out of the total $\mathrm{n}$ genes in the list annotated to that GO term. (G) A zoomed view of the transition profile heatmap restricted to genes from cluster 2 that belong to the "actin binding" GO category. Respective $\operatorname{Tr} 1$ and $\operatorname{Tr} 2$ fold changes are indicated on the heatmap. (H) In situ hybridisation showing that Gel and $d C r y A B$ that are part of the "actin binding" GO class are predominantly expressed in LT muscles. Lateral views of stage 15 embryos are shown. Anti-actin or anti- $\beta 3$ tubulin antibodies are used to reveal muscle pattern. Schemes of muscles in an abdominal segment are shown with Gel and $d C r y A B$ expression indicated by a color code: red - high expression and light pink - low expression levels.

demonstrated that Eve, $\mathrm{Lb}$ and Slou iTFs regulate the number of fusion events by setting expression levels of genes that act as identity realisators in a muscle-specific manner ${ }^{13,15}$. However, the identification of "realisator genes" has so far been limited to only a few examples, owing to the technical challenges of detecting gene expression in specific muscle populations during embryogenesis.

To further analyse diversification processes and identify genes acting downstream of iTFs, we optimised translating ribosome affinity purification (TRAP) ${ }^{16}$ to small subsets of FCs and developing muscle precursors ${ }^{17}$. TRAP-purified mRNA profiling followed by bioinformatic analysis and generation of temporal transition profiles identified muscle subset-specific translatome signatures with Gelsolin (Gel) and $d C r y A B$ as new identity realisator genes controlling shape- and size-related properties of Lms-expressing muscles.

Intriguingly, $d C r y A B$ and $G e l$ act downstream of Ap and Lms and their loss-of-function phenotypes recall dystrophic muscle branching/splitting in humans ${ }^{18}$.

\section{Results}

Translational profiling (TRAP) of muscle subsets identifies $d C r y A B$ and Gelsolin expressed predominantly in LT muscles. TRAP is based on the polysome capture of the GFP-tagged ribosomes with their associated mRNAs (Fig. 1A). Here we tagged polysomes with UAS-Rpl10A-GFP in two muscle subsets using Slou-GAL4 or Lms-GAL4 drivers (Fig. 1B) and in all embryonic muscles using Duf-GAL4. For each muscle subset, translational profiling was performed on embryos collected from three developmental time windows T1: 7-10 h AEL, T2: 10-13 h AEL and T3: 13-16 h AEL covering the main muscle development steps. To assess the specificity of TRAP-based muscle targeting, we analysed GO terms. We found that the up-regulated genes fitted muscle-related GOs while GO categories associated with the list of down-regulated genes were not related to muscle developmental processes (Fig. 1C). Gene expression profiling with total embryonic RNA (input fractions) as a reference was used to identify differential gene expression and perform spatial and temporal gene clustering. A significant portion of upregulated genes $(\mathrm{FC}>2, p<0.05)$ turned out to be common to the restricted muscle populations (Slou- and Lms-positive) and the overall (Duf-positive) population (dark colors in Fig. 1D). The percentage of specific transcripts (light colors) remained relatively constant until the latest time point where the proportion of Lms-specific transcripts increased slightly compared to Slou (29\% versus $23 \%$ respectively ,Fig. 1D). This difference may be due to Lms-expressing muscles being less heterogeneous than Slou -positive ones and expressing specific set of genes for their terminal differentiation. Finally, to test muscle type-specific gene expression, we generated volcano plots (Fig. S1A,B) and observed that genes with previously characterised expression patterns in muscle subsets displayed expected up- or down-regulation. For example, in Lms-positive muscles, ap and lms gene transcripts were enriched, whereas slou and org1 transcripts, specific for Slou-positive muscles, were depleted (Fig. S1B). We also tested whether TRAP would detect "low expression" genes. To do so, we crossed our lists of enriched and depleted transcripts with modEncode datasets ${ }^{19}$ and found that more than $30 \%$ of up-regulated TRAP-ed genes entered the "low expression" modEncode category, whereas most depleted transcripts fitted the "high expression" modEncode category (Fig. S1C,D). TRAP-based translational profiling of muscle subsets was thus specific for the targeted muscle populations and sensitive enough to detect low transcript levels.

We then applied temporal transition profiling to identify clusters of genes showing similar dynamics of expression patterns, thus potentially under common upstream regulatory cues. We considered that this approach could be applied to TRAP datasets to identify novel muscle identity realisator genes acting downstream of iTFs ${ }^{15}$. The generated temporal transition heatmap for Slou- and Lms-positive muscles revealed clusters of genes with several expression behaviours (Fig. 1E).

Here we focused on gene clusters showing two contrasting transition behaviours, "down-down” (Cluster 1) and "up-up" (Cluster 2). Cluster 1 genes were characterised by enrichment of GOs associated with "chromatin binding" (Fig. 1F). Among Cluster 1 genes, we found twist and several Notch pathway-involved genes whose 

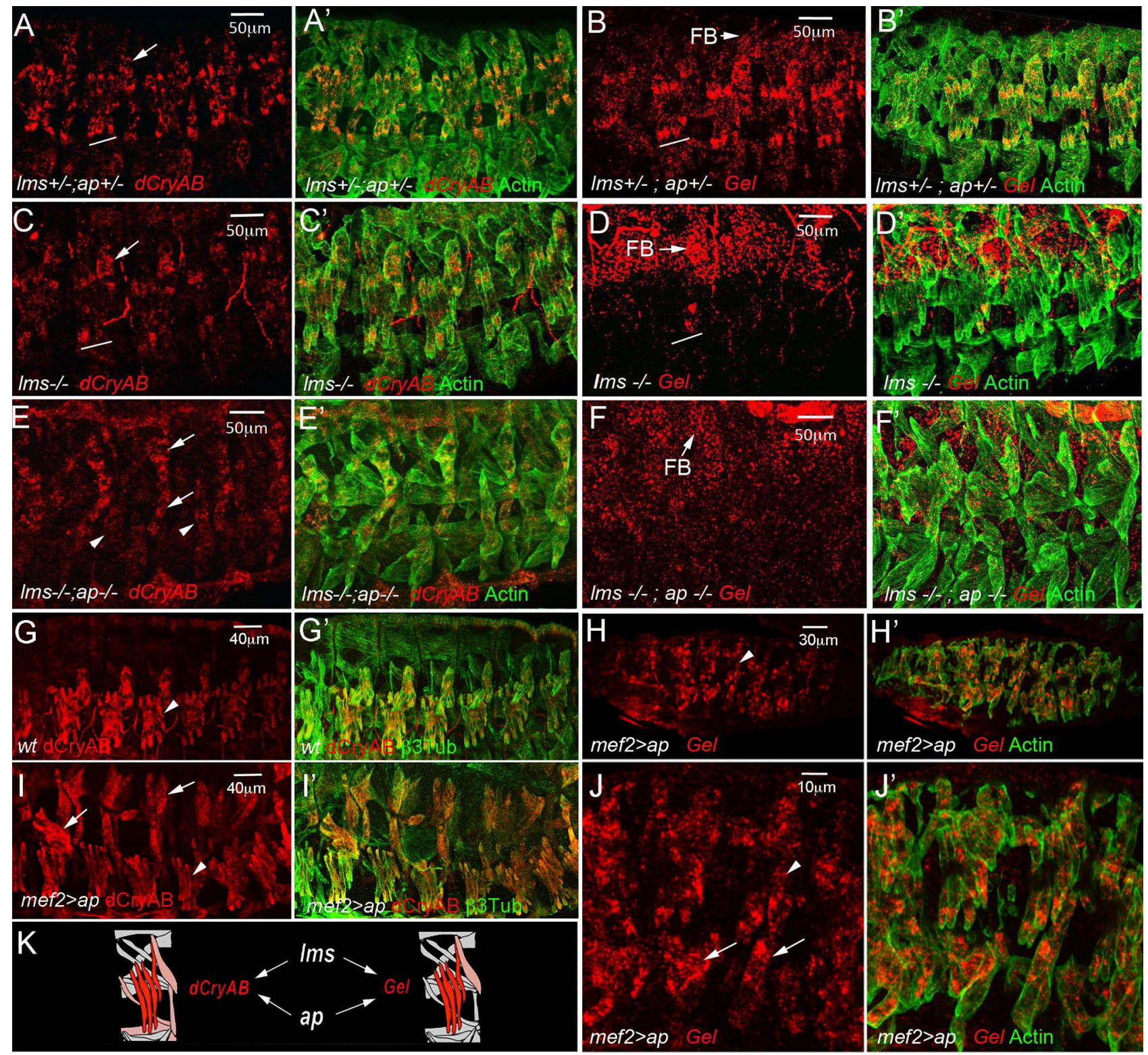

Figure 2. Lms and Ap positively regulate the expression of $d C r y A B$ and $\mathrm{Gel}$ in LT muscles. $d C r y A B$ and $\mathrm{Gel}$ transcript expression were analysed in heterozygous $l m s+/-; a p+/-\left(\mathbf{A}-\mathbf{B}^{\prime}\right)$ embryos, in single $l m s$-/- mutant embryos $\left(\mathbf{C}-\mathbf{D}^{\prime}\right)$ and in double lms-/-;ap-/- mutants $\left(\mathbf{E}-\mathbf{F}^{\prime}\right)$ by in situ hybridisations with $d C r y A B$ or Gel RNA probes. (A-B') Both $d C r y A B$ and $G e l$ are prominently expressed in LT muscles of heterozygous $l m s+/-; a p+/-$ embryos. By contrast, $d C r y A B$ transcript levels are reduced in LTs (underlined area) in simple lms-/- mutants $\left(\mathbf{C}-\mathbf{C}^{\prime}\right)$ compared to $\left(\mathbf{A}, \mathbf{A}^{\prime}\right)$. Note that in $l m s$ mutants, $d C r y A B$ expression in DT1 remains unaffected (arrow). $d C r y A B$ down-regulation in a few remaining LTs is even more apparent (arrowheads) in double lms-/-;ap-/mutants in which $d C r y A B$ expression in SBM and in DT1 is still detectable (arrows). Gel LT expression in the $l m s$-/- context has almost completely vanished (underlined area) in (D) compared with (B), whereas its expression in the fat body (FB) remains high. No LT associated Gel expression could be detected in double lms-/-; ap-/- mutants. Note that Gel expression in FB could still be detected (F). Conversely, pan-muscular expression of ap using Mef2-Gal4 driver induces both dCryAB (compare G, G', with I, I') and Gel expression $\left(\mathbf{H}, \mathbf{H}^{\prime}, \mathbf{J}, \mathbf{J}^{\prime}\right)$ in all muscles. Arrowheads point to LT muscles and arrows point to ectopic expression in dorsal (I) and ventral (J) muscles). (K) Schematic representation of $d C r y A B$ and $G e l$ transcriptional regulation.

expression needs to be turned down while muscle differentiation progresses. By contrast, Cluster 2 showed a significant enrichment of several GO terms related to muscle development (Fig. 1F) including conserved genes encoding actin-binding proteins: CG34417/Smoothelin, flr/WDR1, CG18135/Gpcpd1, sals/SCAF1, Actn/ACTN2, Gel/GSN, TpnC41C/CALM1, dCryAB/l(2)efl/CRYAB (Fig. 1G). We found this sub-cluster of particular interest as it contains genes with similar biological functions and differential Lms- versus Slou- transition profiles. Among them, Gel and $d C r y A B$ with "up-up" transition profile in the Lms subpopulation (Fig. 1G) are both preferentially expressed in Lms-positive LT muscles (Fig. 1H). Drosophila Gel belongs to the conserved Gelsolin/Villin family of 
actin interactors ${ }^{20}$ with actin depolymerisation activity ${ }^{21,22} \cdot d C r y A B$ codes for a small heat shock protein (sHSP) carrying an actin-binding domain and known to interact with cheerio/filamin ${ }^{23}$. Gel transcripts could be detected in LT muscle precursors from late stage 14 (Fig. 1H) but not earlier (Fig. S2A,A'B,B'). Moreover, in late stage embryos $\mathrm{Gel}$ is prominently expressed in the visceral muscles and developing fat body (Fig. S2C,C'). $d C r y A B$ transcripts accumulate preferentially in LTs and in DT1 starting from early embryonic stage 15 (Fig. $1 \mathrm{H}$ ) and at a lower levels in a larger population of lateral and ventral muscles including SBM and VT1 (Fig. 1H, Fig. 2A,A'). Similar to transcripts, both dCRyAB and Gel proteins are detected in LT myotubes with an accumulation at LT extremities and at sub-membrane areas (Fig. S3). LT muscle specification is under the control of identity gene $M s h$ and downstream iTFs, Ap and $\mathrm{Lms}^{6}$. Gel and $d C r y A B$ LT expression (Fig. 2A-B) is dramatically reduced in lms (Fig. 2C-D) and in particular in lms/ap mutant embryos (Fig. 2E-F). Also, both dCryAB and Gel are ectopically activated by panmuscular Ap (Fig. 2G-J') showing that LT iTFs positively regulate Gel and $d C r y A B$ (Fig. 2K).

dCryAB promotes regular shapes and prevents branching of growing LT muscles. To test whether $d C r y A B$ helps set LT muscle features, we generated null allele $\left(d C r y A B^{H R}\right)$ using CRISPR mutagenesis (Fig. 3A). $d C r y A B$ loss-of-function turned out to be homozygous lethal with mutants surviving until the late $3 \mathrm{rd}$ larval instar. Compared to wild-type, the late stage embryos devoid of $d C r y A B$ (Fig. 3B-D) showed dissociations between LT1 and LT2 and/or LT2 and LT3 muscles (32\% of segments) and irregular growth of LTs (28\% of segments). The partial dissociation of LTs was in several instances associated with the irregular LT shapes (Fig. 3B, C) and branched LT muscle extremities ( $8 \%$ of segments) (Fig. 3C, right panel). We also noted a reduced number of LTs in $6 \%$ of segments (Fig. 3D). Branched LT muscles are also occasionally detected in ap and lms mutant contexts (Fig. S4) indicating that dCryAB is involved in preventing LTs branching downstream of LT iTFs.

The irregular LT growth in $d C r y A B$ mutant embryos raised the question of whether $d C r y A B$ could impact on LT interactions with tendon cells and with motor neurons. By the end of embryonic stage 15, $B$-PS integrin accumulates at the extremities of muscles and at the surface of their cognate attachment sites, promoting the formation of myotendinous junctions (MTJs). Accordingly, in wild type stage 15 embryos, ß-PS integrin labels ventral and dorsal ends of LTs (Fig. 3E-E”). However, in $d C r y A B$ mutants this B-PS accumulation was hardly detected, suggesting impaired MTJs formation (Fig. 3F-F"). Because in late $d C r y A B$ mutant embryos we do not detect an LT muscle detachment phenotype, observed in the $\beta$-PS loss-of-function context, we conclude that $d C r y A B$ mutation impairs but does not prevent $\beta$-PS accumulation at LT MTJs. Consistent with this, in 2nd instar $d C r y A B$ mutant larvae, LTs, including those with branched ends, appear attached (Fig. 3G,H).

We then tested whether the LTs in $d C r y A B$ mutants were properly innervated. In wild type embryos, the dorsal branch of the SNa nerve innervating LTs defasciculates at the level of LT2 and grows dorsally within a gap between the ventral extremities of LT2 and LT3 (Fig. 3I-I"). In $d C r y A B$ mutants, most segments have wild type LT innervation, but in those with forked LT ends (essentially seen at the ventral extremity of LT2), the gap between LT2 and LT3 is filled, preventing defasciculation of SNa and LT innervation (Fig. 3J-J").

Thus, by coordinating LT muscle growth, dCryAB ensures timely accumulation of B-PS integrin and optimal LTs attachment. The capacity of dCryAB to control LT shapes and prevent their branching facilitates proper LT innervation.

Gel mutant embryos bear an increased number of LTs through fibre splitting. The CRISPR mutagenesis in the 5' region of Gel resulted in two different null mutations, Gel9.3 and Gel9.8 (Fig. 4A), both leading to a premature stop codon. Because Gel9.3 and Gel9.8 exhibited similar molecular lesions, were homozygous viable, and showed equivalent LT muscle phenotypes (Fig. 4B), we chose one of them, Gel9.3, for further analyses.

Despite irregularity in LT growth (32\% of segments) and some LT dissociation phenotypes (14\% of segments), Gel mutants also showed an increased number of LT muscles (17\% of segments) (Fig. 4B-D), a phenotype previously described (6) and detected in particular in ap mutants (Fig. S4B), however not observed in the $d C r y A B$ loss of function context (Fig. 3).

To follow formation of supplementary LTs in vivo, we recombined Gel9.3 with the Lms > LifeActinGFP (LAGFP) LT sensor line. We first confirmed that Gel9.3;Lms > LAGFP embryos form the supernumerary LTs, which become individualised with connectin-labeled cellular membranes (Fig. 4F,F'). We then performed time lapse experiments encompassing mid-embryogenesis on Lms > LAGFP (Fig. S5A, control) and on Gel9.3;Lms > LAGFP embryos in which splitting occurs (Fig. S5B). The example of splitting presented (Fig. 4E, Fig. S5B) concerns LT3 which grows asynchronously, expands and subdivides progressively into two fibres with separate extremities. This aberrant morphogenesis appears to have a functional impact, as at the beginning of larval life, the striated sarcomeric pattern of split LT muscles is severely impaired (Fig. 4G,G'), indicating that their contractility is compromised. Thus, the supernumerary LTs in Gel mutants arise from enlarged fibres that eventually split. Another, interesting feature is that split LTs extremities accumulate BPS-integrin (Fig. 5A), suggesting that LT identity information ensuring choice of attachment sites is transmitted during splitting.

Gel controls LT muscle size by preventing excessive myoblast fusion. On performing time lapse experiments, we observed that splitting occurred during the developmental period in which muscle fibres grow by fusing with surrounding myoblasts, and that split LTs were enlarged compared to non-split neighbours (Fig. 4E). Our previous findings showing that muscle size depends on the number of fusion events ${ }^{15,24}$ thus raised the question of whether LT splitting could be associated with increased fusion. This appears to be the case since the LT-targeted increase in fusion by overexpressing Duf could lead to splitting (Fig. 5B,C). On the other hand we found that Gel is expressed in developing LT muscles but not in FCs (Fig. S2) and that the number of 
A

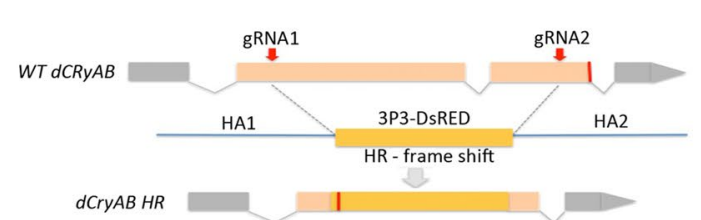

B

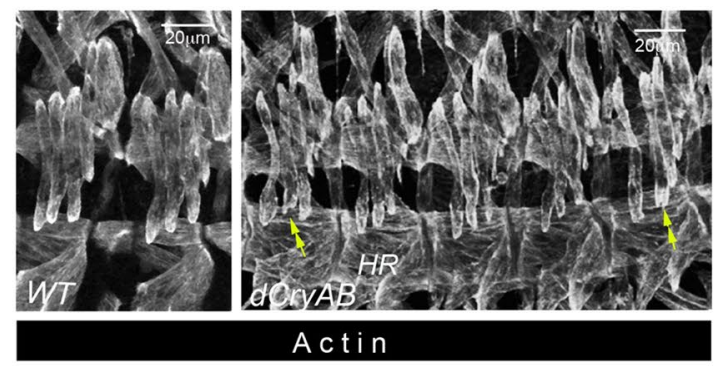

D

D ${ }_{35 \%} \quad$ LT muscles shape phenotypes

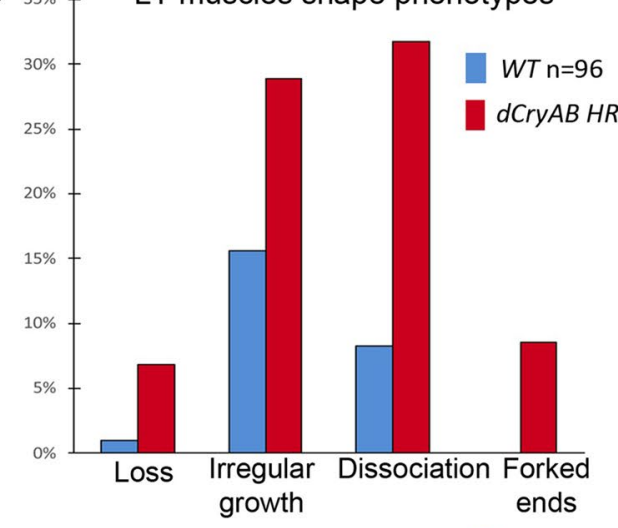

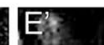
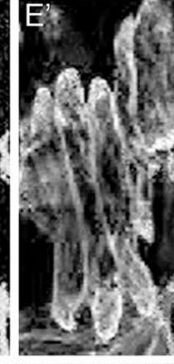

$\$$
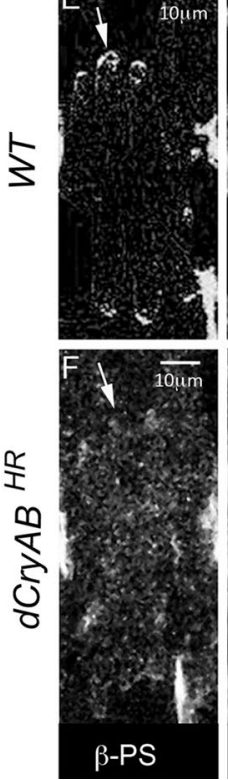
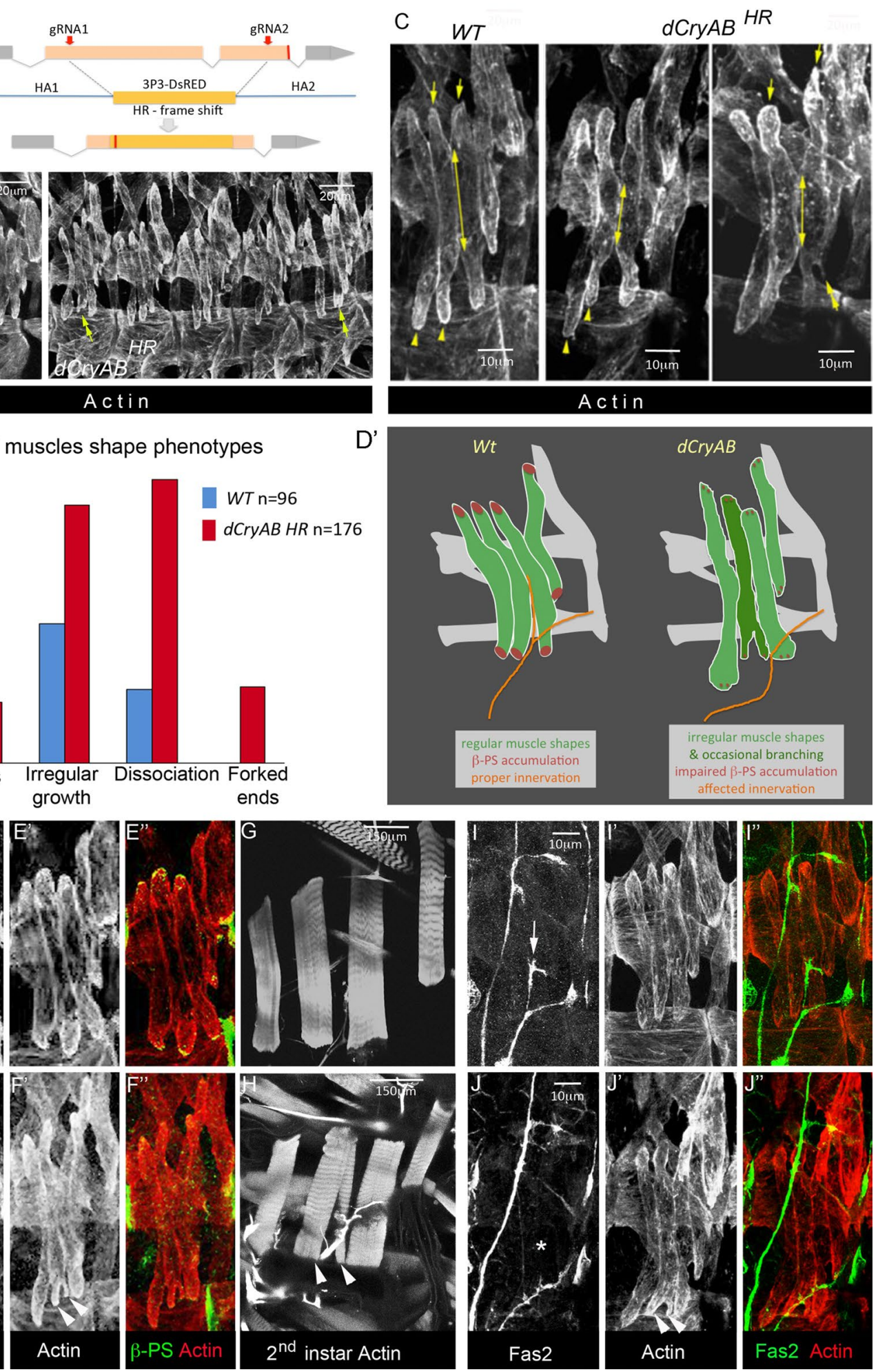

\author{
$\mathrm{D}^{\prime}$
}
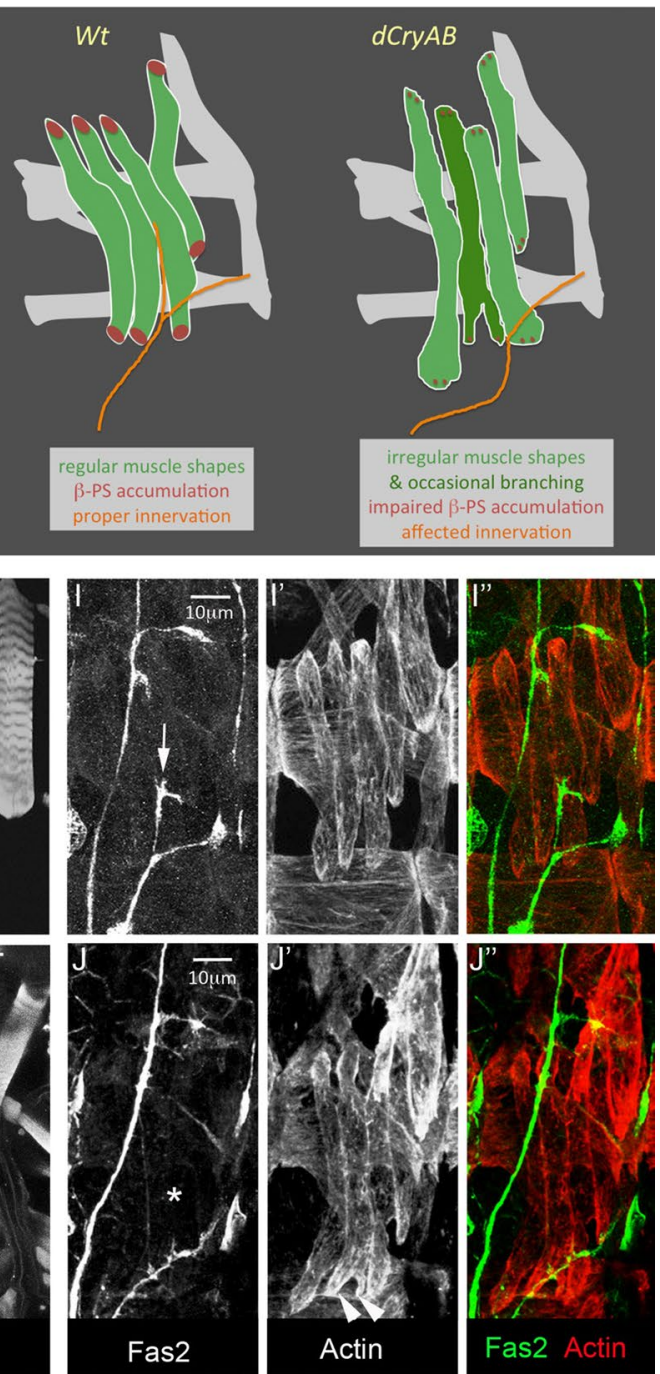
4Figure 3. Aberrant growth-related LT muscle properties in $d C r y A B$ mutant embryos. (A) Schematic representations of CRISPR mutagenesis of $d C r y A B$ locus. gRNAs targeting sites are indicated by red arrows, and positions of resulting stop codon by red line. Homology directed recombination (HDR) with replacement of the $d C r y A B$ coding sequence by a 3P3-dsRed cassette was applied to generate the $d C r y A B^{H R}$ null mutant line. (B-C) Representative lateral views of muscles from late stage 15 embryos stained with anti-actin antibody to reveal muscle shapes. Yellow arrows and yellow arrowheads point to dorsal and ventral LT termini, respectively. They are aligned in wild type but appear at different levels in two neighboring LTs in $d C r y A B^{R H}$, suggesting irregular LT growth. Double-headed arrows show extents of contact between LT2 and LT3 muscles at the end of stage 15 , which are often reduced in the $d C r y A B^{R H}$ mutant context, indicating partial dissociation of growing LTs. Double-headed arrowhead points to forked/branched LT ends. (D) Quantification of LT muscle phenotypes in $d C r y A B^{R H}$ embryos presented as percentage of segments affected ( $n$ - number of abdominal segments examined). ( $\left.\mathbf{D}^{\prime}\right)$ A scheme representing LT muscle defects in $d C r y A B$ loss of function context. (E-F"). Lateral view of late stage 15 embryos, showing reduced B-PS Integrin staining at LT muscle termini in the $d C r y A B^{R H}$ mutant context (F-F") compared to WT embryo (E-E"). (G-H) Lateral view of $2^{\text {nd }}$ instar $d C r y A B^{R H}$ larvae, showing forked end phenotype on LT2 muscle (arrowheads in H). (I-J") Lateral view of late stage 15 embryos, stained for fasciclin 2 to show aberrant defasciculation of SNa motoneuron in $d C r y A B^{R H}$ context (compare arrow (I) and star in $\mathbf{J})$.

LT FCs in Gel mutants remains unchanged (Fig. 5D), suggesting that the supernumerary Gel-devoid LTs arise by an aberrant fusion-involving muscle morphogenesis.

Indeed, the number of Mef2-positive nuclei in LT1-LT4 at embryonic stage 16 was significantly higher in $\mathrm{Gel}$ mutants including the transheterozygous Gel9.3/Gel9.8 context compared to controls (Fig. 6A-D). We then sought to determine whether the LT-specific increase in fusion events observed in Gel mutants could impact on the fusion programs of neighbouring muscles. The number of myonuclei in immediate LT neighbours in the SBM and LO1 muscles (but not in more ventrally located VT1) was reduced, indicating that local availability of FCMs could impact on fusion programs (Fig. 6E,F). Muscle splitting observed in Gel mutants thus results from excessive fusion, with late fusion events that could be detected associated with LTs showing a split phenotype (Fig. 6B, right panel). The capacity of $\mathrm{Gel}$ to negatively regulate fusion was confirmed by the reduced number of myonuclei in LTs in which Gel was prematurely activated (Fig. 6D) and by the large number of unfused myoblasts seen in embryos with ectopic Gel expression in all muscles (Fig. 6G). Thus, we propose that Gel triggers fusion arrest in LTs. In Gel loss of function context LTs continue to grow by fusion and eventually split (Fig. 6H).

\section{Discussion}

TRAP was first developed to isolate polysome-associated mRNA from a subset of neurons in mice ${ }^{16}$ and was later adapted to other model organisms including Xenopus ${ }^{25}$, zebrafish ${ }^{26}$ and Drosophila ${ }^{17,27}$. Here we applied TRAP to determine the first translatomic signatures underlying diversification of muscle types, and we identified $\mathrm{Gel}$ and $d C r y A B$ as new LT muscle identity realisator genes. $d C r y A B$ contribute to preventing LTs branching, and $\mathrm{Gel}$ plays a role in LT muscle size control by limiting the number of fusion events. Consequently, supernumerary myonuclei are present in Gel-devoid LTs, which eventually split. Both splitting and branching are low penetrance phenotypes (17\% and $8 \%$ of segments, respectively), indicating that Gel and $d C r y A B$ are not the sole identity realisators that prevent these aberrant growth-related LTs features.

Formation of branched muscle fibres has recently been reported as a result of adversely affected muscle identity ${ }^{28}$, and supernumerary LTs were also detected in $a p$ and $l m s$ mutant embryos ${ }^{6}$. Here we report that split and branched muscles are occasionally detected in both LT iTF and Gel or $d C r y A B$ mutant embryos indicating that a muscle identity-dependent shape and size control system operates in developing muscles.

In humans, $C r y A B$ mutations are associated with desminopathies in which aberrant muscle fibres with branched morphology are frequently detected ${ }^{29}$. Gelsolin mutations cause amyloidosis, characterised by the toxic accumulation of protein aggregates, which can lead to an inclusion body myositis (IBM)-like phenotype with necrotic and centronuclear split fibres ${ }^{30,31}$.

Functional analyses of $d C r y A B$ and $G e l$ in Drosophila embryos indicate that muscle fibres branch or split when the identity realisators for these muscle are not properly activated.

Reduced levels of $B$-PS integrin at the extremities of $d C r y A B$-devoid LTs suggests weak interactions with attachment sites and could explain observed LTs overgrowth and dual attachment of branched fibers. These $d C r y A B$ loss of function phenotypes could result from inappropriate actin cytoskeleton dynamics in growing LTs and/or affected function of cheerio/filamin, a direct dCryAB interactor ${ }^{23}$. The observation that dCryAB protein accumulates at sub-membrane areas and at LT myotube ends suggests it could contribute to preventing non-polarized, branched LT growth.

In advanced stages of muscular dystrophies, a large subset of muscle fibers shows longitudinal splitting, described already more than forty years ago (34). After chemical or physical injury, split fibers form also in undergoing regeneration wild type muscles (35) suggesting a link between myoblast fusion-involving regeneration (chronic in dystrophic context) and muscle splitting. However, despite critical impact on dystrophic muscle cytoarchitecture, on vulnerability to contraction-induced damage and on muscle weakening, mechanisms of muscle fibers splitting remain unexplored $(36,37)$.

Our finding in Drosophila that local increase in fusion occuring in Gel mutant embryos causes split fiber phenotype points to the excessive fusion as a prerequisit of splitting. Because Gel protein displays actin-severing properties and as we show here partially colocalises with actin in growing LTs, we speculate it could affect submembrane F-actin sheet and reduce the required for fusion myotube rigidity. 

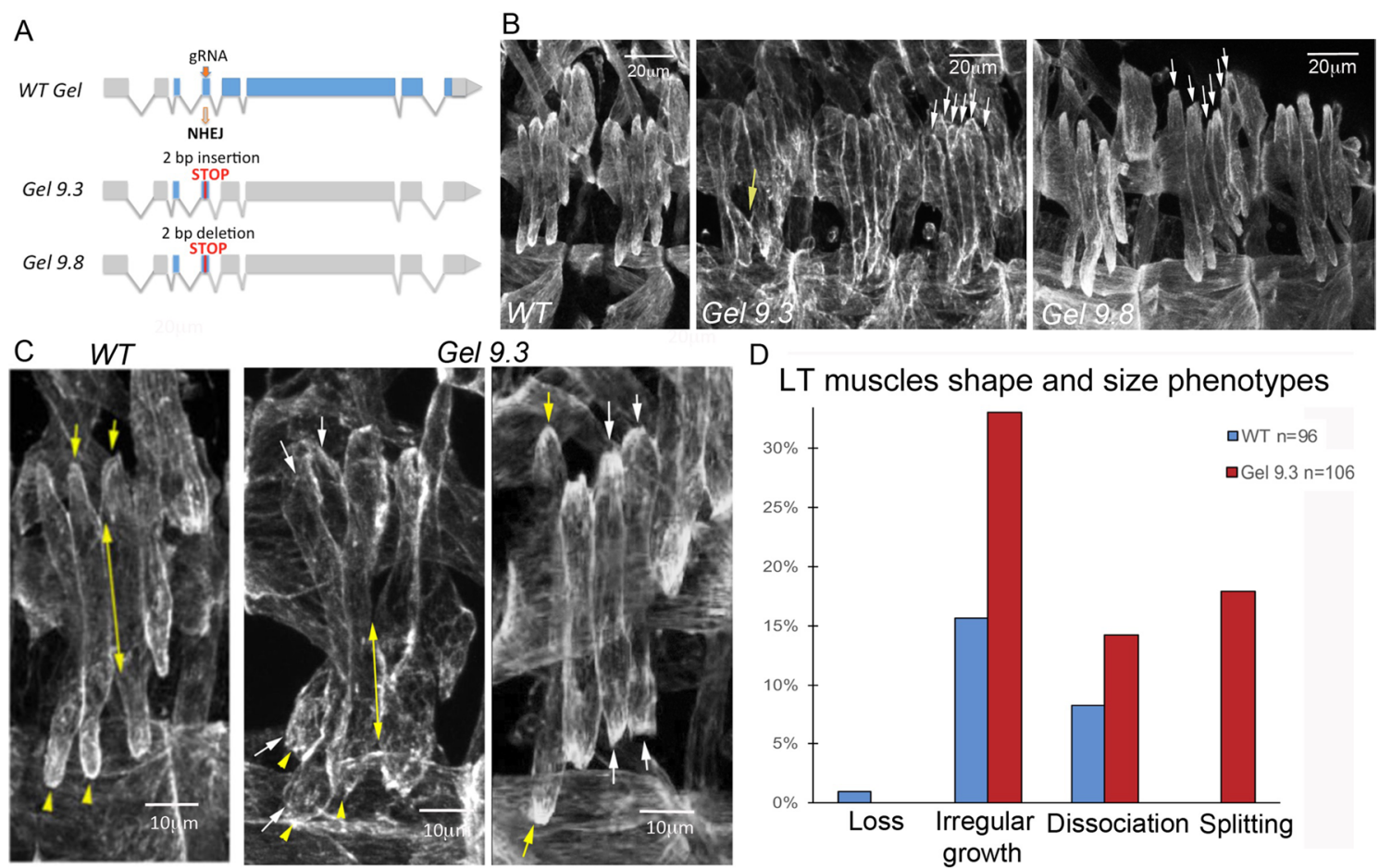

${ }^{\mathrm{D}}$ LT muscles shape and size phenotypes
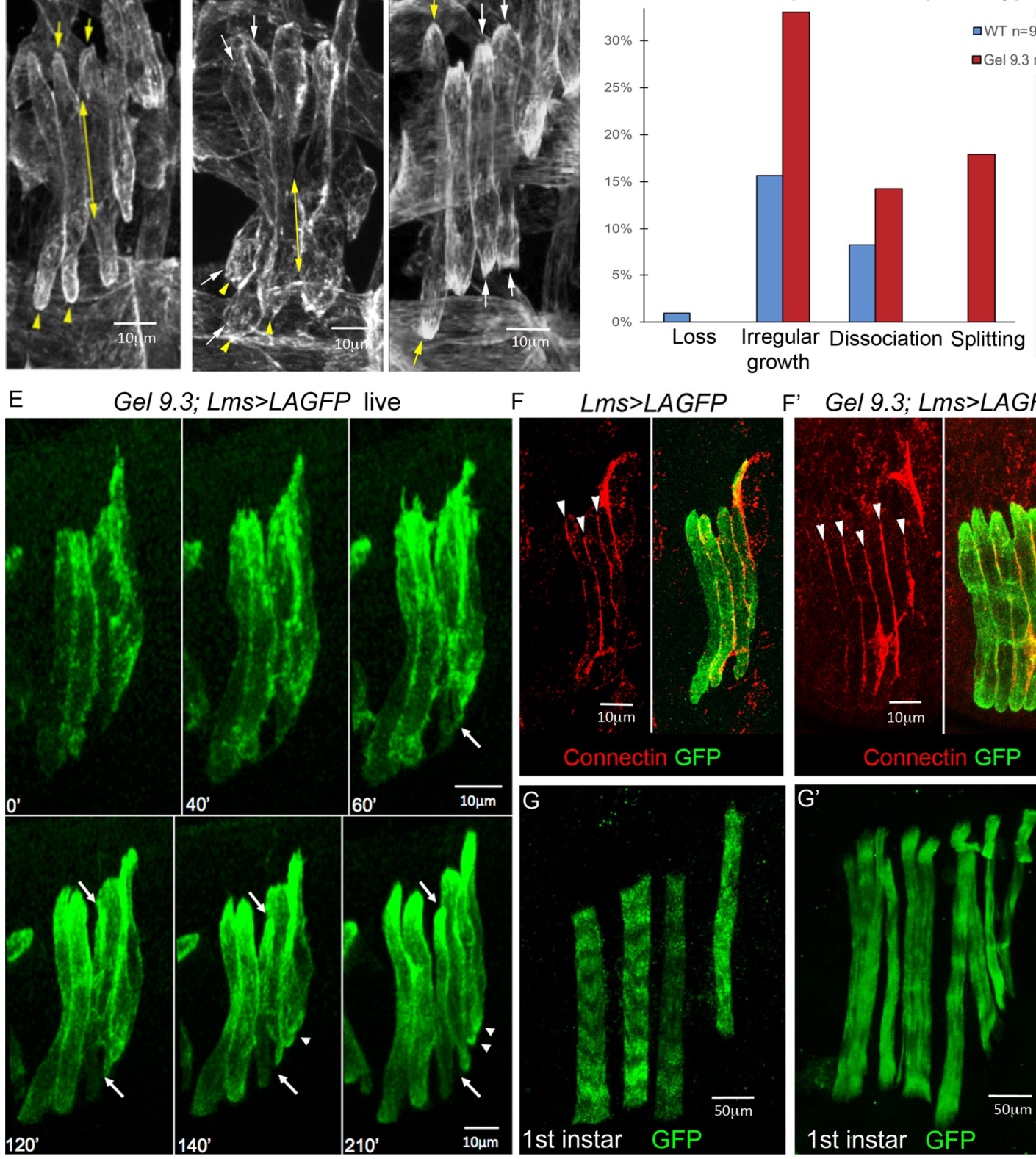

F $\quad L m s>L A G F P$

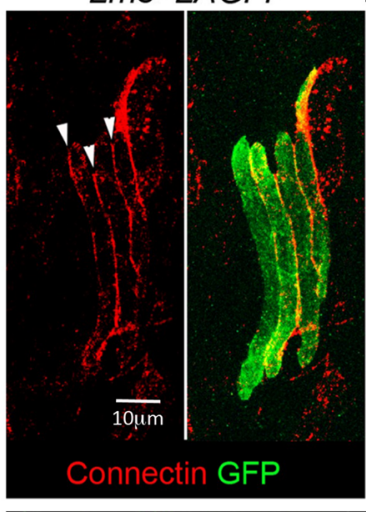

G

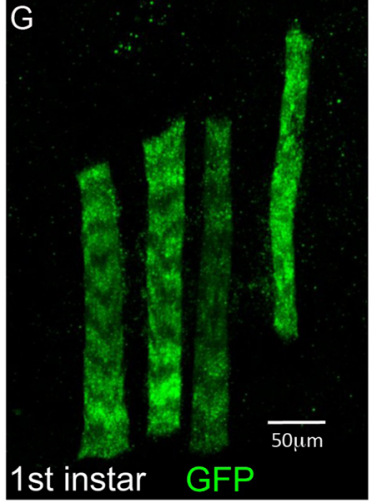

F' Gel 9.3. Lms>LAGFP
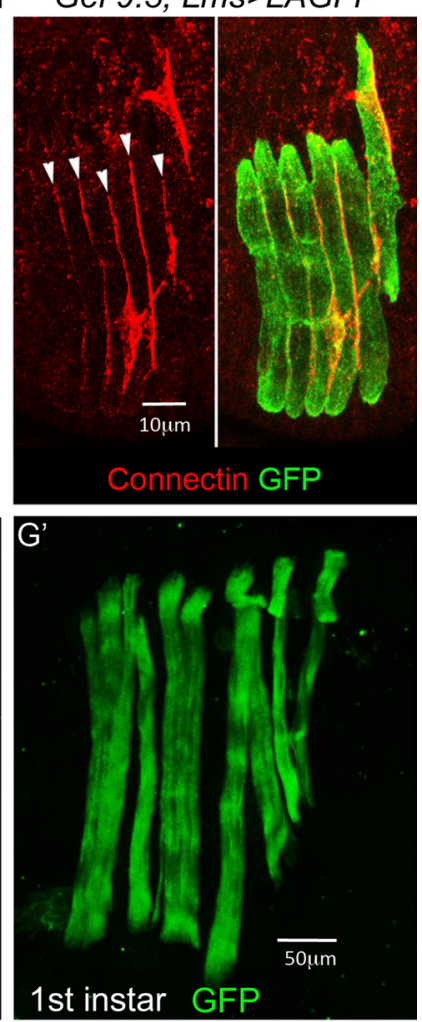
4Figure 4. Gel mutant embryos show LT muscle splitting. (A) Schematic representations of CRISPR mutagenesis of $\mathrm{Gel}$ locus. The gRNA targeting site is indicated by red arrow and positions of resulting stop codons by red lines. Gel 9.3 mutant was generated by a 2 bp insertion and Gel 9.8 mutant by 2 bp deletion in the 5' region of the Gel locus. $(\mathbf{B}, \mathbf{C})$ Representative lateral views of muscles from late stage 15 embryos stained with anti-actin antibody. Upper panels (B) show two wild type abdominal hemisegments and three hemisegments from $\mathrm{Gel} 9.3$ and $\mathrm{Gel} 9.8$ mutants. White arrows point to excessive LT muscle extensions and the yellow arrow shows dissociation phenotype. Lower panels (C) are the zoomed views of LT muscles. Yellow arrows and yellow arrowheads point to dorsal and ventral LT termini, respectively. Double-headed arrows show extents of contact between LT2 and LT3 muscles at the end of stage 15, often reduced in the Gel 9.3 mutants. White arrows point termini of muscles showing splitting phenotypes. (D) Quantification of LT muscle phenotypes in Gel 9.3 embryos presented as percentage of segments affected ( $n$ - number of abdominal segments examined). (E) Snapshots over time of LT muscle shapes in Gel 9.3 mutants revealed using the Lms $>$ LifeActin-GFP (Lms $>$ LAGFP) LT sensor line. After $60 \mathrm{~min}$, a first separation of muscle is observed (white arrow) on the ventral side of the LT3 muscle. This event is followed by actin cytoskeleton changes leading to progressive accumulation of actin on the dorsal side (upper white arrow) dissociation from LT2 muscle and individualisation of a new muscle fibre. Arrowheads show additional partial splitting of LT4 muscle on its ventral side. $\left(\mathbf{F}-\mathbf{F}^{\prime}\right)$ Connectin-stained LT fibres in wild type and in gel mutants. Note increased number of connectin lines (arrowheads) in the segment with split LT muscles in the gel context (F) compared to the Lms $>$ LAGFP context (F). (G-G') Lateral view of 1st instar larvae, showing split LT muscles with disorganised sarcomeric structures in the $\mathrm{Gel} 9.3$ context.

\section{Materials and methods}

Fly stocks. All D. melanogaster stocks and crosses were grown on standard medium at $25^{\circ} \mathrm{C}$. The following strains were used: $a p^{U G O 35}$ (gift of J. Botas, Baylor, Houston, USA), $l m s^{95}$ (gift of D. Müller, Universität Erlangen-Nürnberg, Germany), UAS-LifeAct-GFP (UAS-LAGFP) (Bloomington, 35,544) and UAS-Dumbfounded (UAS-Duf) (gift of M. Ruiz-Gomez, Spanish National Research Council, Spain). To generate the TRAP lines, the UAS-RpL10a-EGFP line was crossed with the Slou-Gal4 (gift of M. Frasch, Universität Erlangen-Nürnberg, Germany), Lms-Gal4 (Janelia Farm collection, 46,861) and Duf-Gal4 (gift of K. Vijayraghavan, TIFR, India) driver lines to specifically target polysomes in the respective muscle populations.

Generation of Gel and dCryAB knock-out lines by CRISPR-Cas9. The different gRNAs were designed using CRISPR optimal target finder ${ }^{32}$. To generate gel guide we used the pair of primers 5'-GTCGAG ACCTCGACCGATGAGGC-3' and 5'-AAACGCCTCATCGGTCGAGGTCTC-3'. The primers were annealed, digested by Bbs 1 and cloned into pCFD3 plasmid (plasmid \#49,410, Addgene) for Gel. For $d C r y A B$ a HDR-based CRISPR technology was applied. First, two guides targeting 5'-CTTGGACCAGCACTTCGGTC-3' and 5'-GGA GGACAACGCCAAGAAGG-3' sequences located in the 5' and 3' regions of the $d C r y A B$ gene, respectively, were designed and cloned by Gibson assembly into pCFD4 plasmid (\#49,411). The homology arms HA1 of 1045 bp and HA2 of 1058 bp were amplified using the following pairs of primers:

Forward HA1: 5'-ATATCACCTGCATATTCGCAGCGACGTCATCTCTTTCGTCTG-3'.

Reverse HA1: 5'-ATATCACCTGCATATCTACAAGAGGCGCGAGGTGCGCATTG-3'.

Forward HA2 : 5'-ATATGCTCTTCATATAGGTGGAGACCTCCACCGCC-3'.

Reverse HA2 : 5'-ATATGCTCTTCAGACTTCGTCAGGTTCGGTTACTCCG-3' into AarI and SapI cloning sites of donor pHD-DsRed plasmid $(\# 51,434)$. Plasmids were injected into Nos-Cas 9 embryos (BestGene).

To establish KO lines, molecular characterisation of target loci was performed as described ${ }^{33}$. Briefly, genomic DNA was extracted from individual larvae by crushing them in $20 \mu \mathrm{L}$ QuickExtract solution (Cambio) and releasing the DNA in a thermomixer according to the supplier's instructions. We used $1 \mu \mathrm{L}$ (previously diluted five times) of the supernatant in $25 \mu \mathrm{L}$ PCR reactions. PCR products were then sequenced by Sanger. Indels can be observed as regions with double peaks in heterozygous flies, corresponding to wild type and mutated allele respectively. In the case of $d C r y A B$, homologous recombination events were recovered by selecting flies with red eye fluorescence.

RNA extraction and RT-qPCR. mRNA was extracted from Lms, Slou and Duf muscle populations using TRIzol reagent (Invitrogen) following the manufacturer's instructions. RNA quality and quantity were then assessed using Agilent RNA 6000 Pico kit on Agilent 2100 Bioanalyzer (Agilent Technologies).

Microarray analysis. Agilent $8 \times 60 \mathrm{~K}$ probe $(60$-mer) gene expression microarrays were used. We assessed the quality of triplicates using Pearson's correlation test. Correlations for all conditions were $85 \%$ or higher. The microarray data were quantile-quantile normalised. Gene expression data from Slou-, Lms- and Duf-positive cells were compared to the whole embryo datasets to generate lists of genes differentially expressed, fold change $\geq 2, p<0.05$. GO Princeton software was used to assign GO classification. We then compared Lms- and Slou-positive cells to Duf-expressing cells to make two lists of differentially regulated muscle-specific genes, fold change $\geq 2, p<0.05$. We computed and compared GO biological processes from these two lists using an $\mathrm{R}$ package cluster profiler.

Temporal transition heatmap. Translational temporal profiles from Slou and Lms at the three time points were converted to "transition values" defined as log ratios between T2 and T1, T3 and T2. Transition 
A

Lms>LAGFP; Gel 9.3

B

Lms>LAGFP;UAS Duf
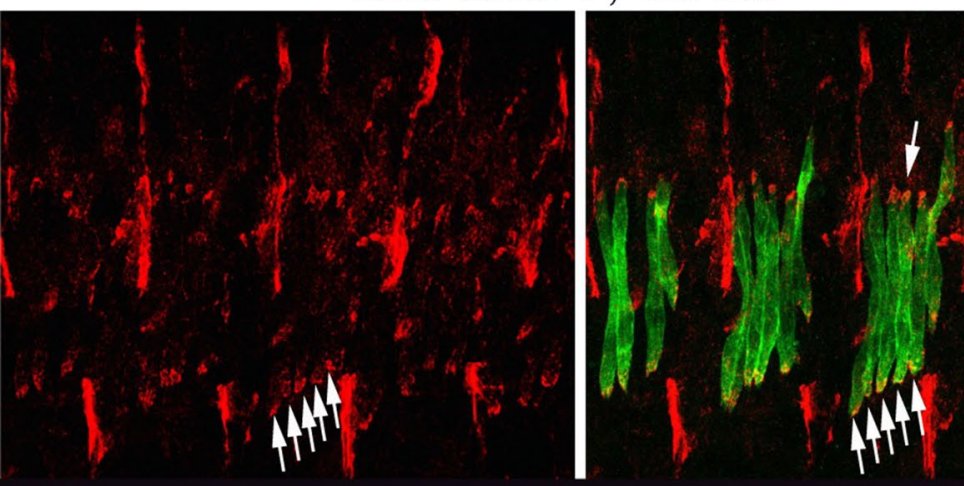

$\left(\begin{array}{l}x \\ +3\end{array}\right.$

GFP BetaPS Integrin

C
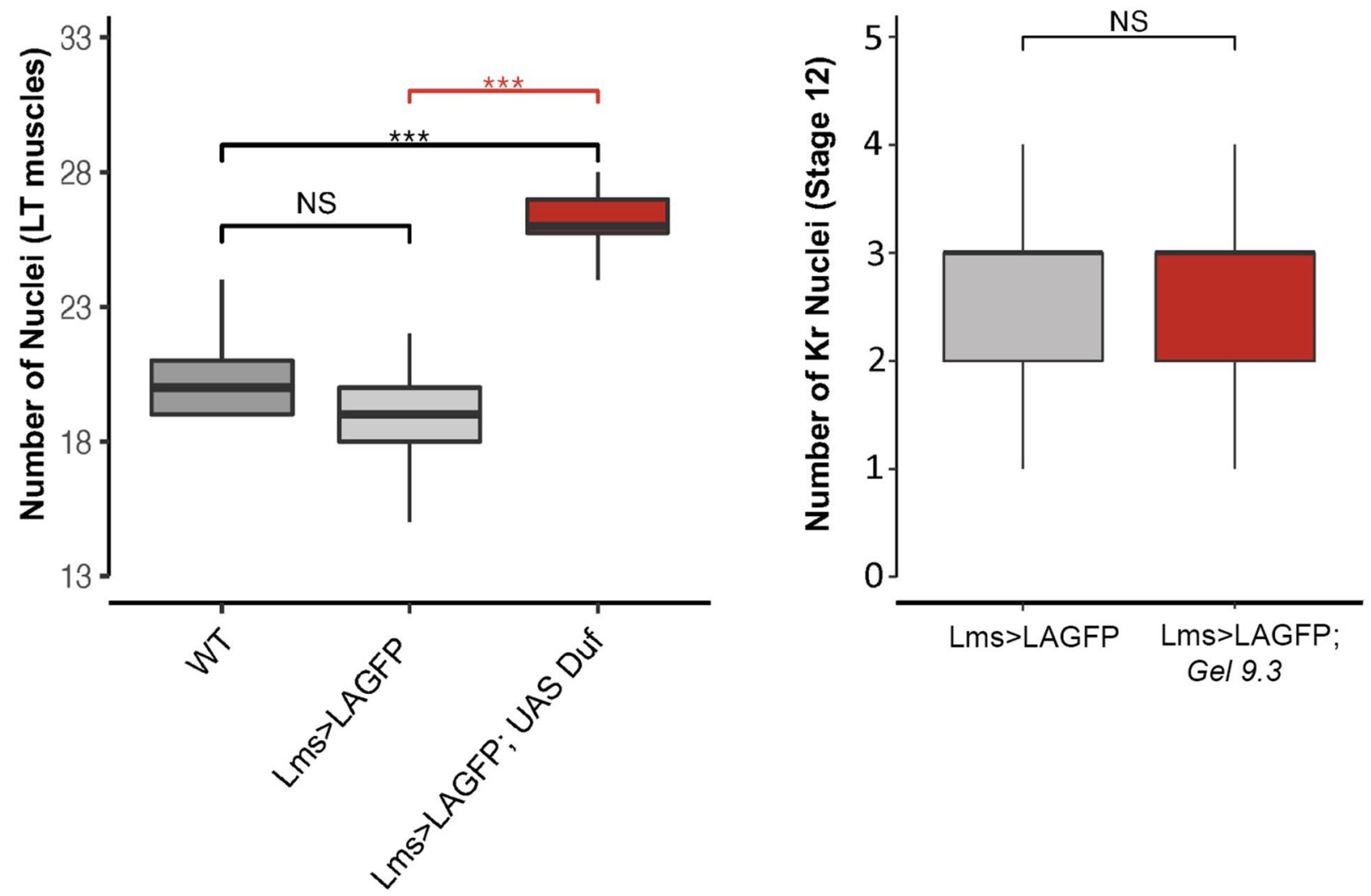

Figure 5. Split LT fibers keep LT properties but do not arise from an excess of progenitors and could be generated by local increase of myoblast fusion. (A) Splitting in Gel mutants is associated with accumulation of ßPS integrin (arrows) at termini of split LT fibres. (B-C) Duf overexpression mimics the Gel LT splitting phenotype (arrows in B), which correlates with increased numbers of myonuclei. Myonuclei were counted (C) in 13 wild type abdominal segments and in $13 \mathrm{Lms}>L A G F P>D u f$ abdominal segments with splitting events and in a larger number of abdominal segments $(n=43)$ from $L m s>L A G F P$. A Wilcoxon-Mann-Whitney test was applied to assess statistical significance. ${ }^{* *} p<0.001$. (D) Graphical representation of number of Kr progenitors at stage 12 showing that supernumerary LT muscle fibres are not due to an excess of LTs progenitors. A large number of hemisegments in Lms $>\operatorname{LAGFP}(n=150)$ and Gel 9.3;Lms $>$ LAGFP mutant embryos $(n=114)$ were analysed. A Wilcoxon-Mann-Whitney test was applied to assess statistical significance. ${ }^{* *} p<0.001$. 
A

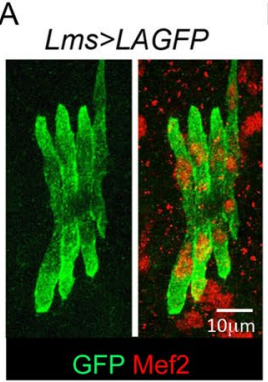

B

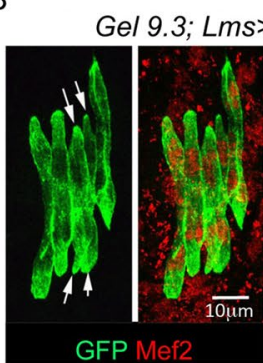

C

E

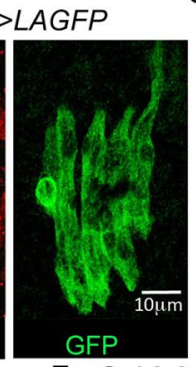

Gel 9.3/9.8

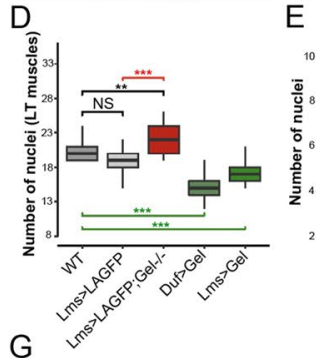

F Gel 9.3; Lms>LAGFP
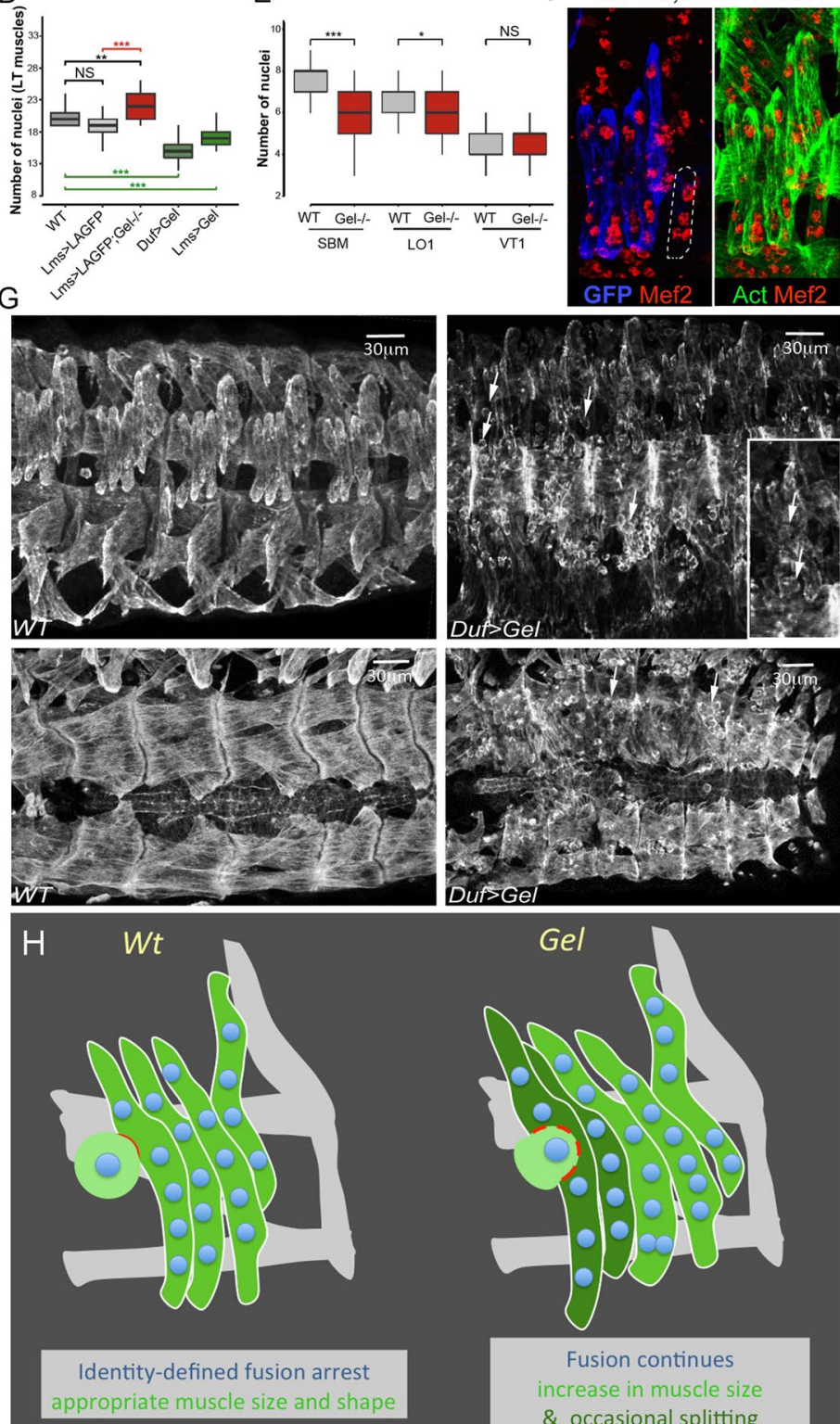

Dut>Ge
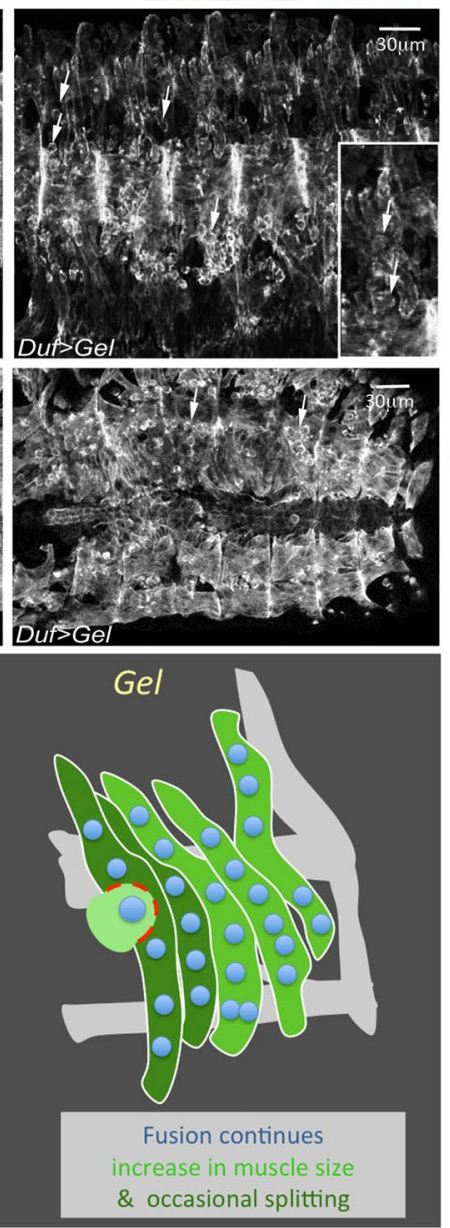

Figure 6. LT muscle splitting in Gel mutant embryos is due to an excessive myoblast fusion. LT muscle shapes at late stage 15 revealed by anti-GFP staining in Lms > LAGFP sensor line (A), in Gel 9.3 context (B) and in transheterozygous Gel 9.3/9.8 embryos (C). Myonuclei are visualised using anti-Mef2. Arrows indicate LT splitting in $\mathrm{Gel} \mathrm{homozygous} \mathrm{(B)} \mathrm{and} \mathrm{the} \mathrm{transheterozygous} \mathrm{context}$ (C). Late fusion events are observed in Gel 9.3;Lms $>$ LAGFP embryos (B, right panel). (D) Number of Mef2 positive LT1-LT4 nuclei is increased in Gel mutants $(n=30)$ compared to wild type $(n=13)$ and $L m s>L A G F P$ contexts $(n=43)$. An opposite effect (reduced number of nuclei) is observed in LTs of Duf $>\mathrm{Gel}(n=80)$ or Lms $>$ Gel embryos $(n=65)$ (green boxplots). A Wilcoxon-Mann-Whitney test was applied to assess statistical significance. ${ }^{* *} p<0.001$ (E). Excessive fusion in Gel mutant with split LTs induce a decrease in the number of nuclei in neighbouring SBM and LO1 but not in ventrally located VT1 muscle. For SBM: WT $(n=66)$, Gel $9.3(n=110)$, for LO1: WT $(n=58)$, Gel $9.3(n=100)$, for VT1: WT $(n=68)$, Gel $9.3(n=93)$. A Wilcoxon-Mann-Whitney test was applied to assess statistical significance. ${ }^{* *} p<0.001$. (F) Reduced number of nuclei in SBM muscle (dotted line) of Gel 9.3 embryo (four instead of seven in the wild type). (G) Lateral and dorsal views of anti-actin stained stage 16 wild type and Duf $>$ Gel embryos (right panels) showing myoblast fusion defects. Arrows point to unfused myoblasts. (H) A scheme of Gel loss of function phenotypes. 
values were considered as three discrete classes: upregulated $(>1)$, stable (between -1 and 1 ), and downregulated $(<1)$. Thus expression profiles from Lms and Slou muscles, which contain three temporal windows, were converted into vectors of two transitions $(\operatorname{Tr} 1, \operatorname{Tr} 2)$, which allow the determination of correct gene behaviour. For example, the profile "red-red" group genes whose RNA level increases between T1 and T2 (Tr1), and then continues to increase between $\mathrm{T} 2$ and $\mathrm{T} 3$ (Tr2).

In situ hybridisation and immunostaining. Embryos were dechorionated and fixed in $4 \%$ paraformaldehyde/heptane for all immunohistochemistry. Fluorescent in situ hybridisation with a TSA amplification system (Perkin-Elmer) and immunohistochemistry was as described previously ${ }^{13}$.To generate the RNA probe for Gel (primers used: 5'-5'AATCGACTCCGTGGTGACTC-3' and 5'-GGGAGGCCAAAGATGAGCTGTC-3') the corresponding DNA sequences were cloned by PCR in pCR II topo vector. The corresponding anti-sense RNAs were transcribed in vitro using T7 or SP6 RNA polymerase. For $d C r y A B$, Gold collection clone GH01960 was used to generate RNA probes. For fluorescent staining, the following antibodies were used: rabbit anti- $\beta 3$ tubulin (1:5000; R. Renkawitz-Pohl, Philipps University, Marburg, Germany), rat anti-actin (1:300, MAC 237; Babraham Bioscience Technologies), rabbit anti-Mef2 (Nguyen HT, 1:2000), rabbit anti-dCryAB (1:500) ${ }^{23}$, rabbit anti-Gel (1:20), M. Leptin, EMBL, Heidelberg, Germany), anti-GFP (1:1000 Developmental Studies Hybridoma Bank (DSHB)), mouse anti-Integrin $\beta$ PS (1:200 DSHB) and mouse anti-connectin (1:200 DSHB). Cy3, Cy5, and 488 conjugated secondary antibodies were used (1:300; Jackson Immuno-Research). Embryos were mounted in antifade Fluoromount-G reagent (Southern Biotech). Labelled embryos were analysed using an Leica SP8 confocal microscope equipped with a HyD detector and a 40X objective. Images were processed with ImageJ.

TRAP experiment. RPL10aGFP-tagged embryos were collected, and messenger RNAs from the different muscle populations isolated as described ${ }^{17}$. Micro-array data generated in this study were deposited to GEO database : GSE137443: GSM4079386 to GSM4079439.

Live imaging. The Lms-Gal4; UAS-lifeActGFP (Lms $>$ LAGFP) double transgenic line was generated and used for time lapse imaging of LT muscle formation in the gel mutant context. Image acquisition was performed on manually aligned living embryos at $21^{\circ} \mathrm{C}$ using an inverted Leica SP8 confocal microscope. The time interval between acquisions was set to $3 \mathrm{~min}$ and the acquisition time was 3-4 h. Movies were generated and analysed using Imaris software (Bitplane).

Received: 4 January 2021; Accepted: 3 June 2021

Published online: 23 June 2021

\section{References}

1. Baylies, M. K., Bate, M. \& Gomez, M. R. Myogenesis: a view from Drosophila. Cell 93, 921-927 (1998).

2. Landgraf, M., Bossing, T., Technau, G. M. \& Bate, M. The origin, location, and projections of the embryonic abdominal motorneurons of drosophila. J. Neurosci. 17, 9642-9655 (1997).

3. Rushton, E. et al. Mutations in a novel gene, myoblast city, provide evidence in support of the founder cell hypothesis for Drosophila muscle development. Development 121, 1979-1988 (1995).

4. Carmena, A. et al. Combinatorial signaling codes for the progressive determination of cell fates in the Drosophila embryonic mesoderm. Genes Dev 12, 3910-3922 (1998).

5. Tixier, V., Bataillé, L. \& Jagla, K. Diversification of muscle types: Recent insights from Drosophila. Exp. Cell Res. 316, 3019-3027 (2010).

6. Müller, D. et al. Regulation and functions of the lms homeobox gene during development of embryonic lateral transverse muscles and direct flight muscles in drosophila. PLoS ONE 5, e14323 (2010).

7. D’Alessio, M. \& Frasch, M. Msh may play a conserved role in dorsoventral patterning of the neuroectoderm and mesoderm. Mech. Dev. 58, 217220-217231 (1996).

8. Knirr, S., Azpiazu, N. \& Frasch, M. The role of the NK-homeobox gene slouch (S59) in somatic muscle patterning. Development 126, 4525-4535 (1999).

9. Houweling, A. C. et al. Gene and cluster-specific expression of the Iroquois family members during mouse development. Mech. Dev. 107, 169-174 (2001).

10. Kelly, R. G., Jerome-Majewska, L. A. \& Papaioannou, V. E. The del22q11.2 candidate gene Tbx1 regulates branchiomeric myogenesis. Hum. Mol. Genet. 13, 2829-2840 (2004).

11. Nathan, E. et al. The contribution of Islet1-expressing splanchnic mesoderm cells to distinct branchiomeric muscles reveals significant heterogeneity in head muscle development. Development 135, 647-657 (2008).

12. Busser, B. W. et al. Molecular mechanism underlying the regulatory specificity of a Drosophila homeodomain protein that specifies myoblast identity. Development 139, 1164-1174 (2012).

13. Junion, G. et al. Genome-wide view of cell fate specification: ladybird acts at multiple levels during diversification of muscle and heart precursors. Genes. Dev. 21, 3163-3180 (2007).

14. Schaub, C., Nagaso, H., Jin, H. \& Frasch, M. Org-1, the Drosophila ortholog of Tbx1, is a direct activator of known identity genes during muscle specification. Development 139, 1001-1012 (2012).

15. Bataillé, L. et al. Downstream of identity genes: muscle-type-specific regulation of the fusion process. Dev. Cell 19, 317-328 (2010).

16. Heiman, M. et al. A translational profiling approach for the molecular characterization of CNS cell types. Cell 135, 738-748 (2008).

17. Bertin, B. et al. TRAP-rc, Translating Ribosome Affinity Purification from Rare Cell Populations of Drosophila Embryos. $J$ Vis Exp 103, 52985 (2015).

18. Chan, S. \& Head, S. I. The role of branched fibres in the pathogenesis of Duchenne muscular dystrophy: Fibre branching in muscular dystrophy. Exp. Physiol. 96, 564-571 (2011).

19. Roy, S. et al. The modENCODE consortium, identification of functional elements and regulatory circuits by drosophila modENCODE. Science 330, 1787-1797 (2010).

20. Nag, S., Larsson, M., Robinson, R. C. \& Burtnick, L. D. Gelsolin: the tail of a molecular gymnast. Cytoskeleton 70, 360-384 (2013). 
21. Chellaiah, M. et al. Gelsolin deficiency blocks podosome assembly and produces increased bone mass and strength. J. Cell Biol. 148, 665-678 (2000).

22. Lu, M., Witke, W., Kwiatkowski, D. J. \& Kosik, K. S. Delayed retraction of filopodia in gelsolin null mice. J. Cell Biol. 138, 1279-1287 (1997).

23. Wojtowicz, I. et al. Drosophila small heat shock protein CryAB ensures structural integrity of developing muscles, and proper muscle and heart performance. Development 142, 994-1005 (2015).

24. Tixier, V. et al. Glycolysis supports embryonic muscle growth by promoting myoblast fusion. Proc. Natl. Acad. Sci. 110, 18982-18987 (2013).

25. Watson, F. L. et al. Cell type-specific translational profiling in the Xenopus laevis retina. Dev. Dyn. 241, 1960-1972 (2012).

26. Tryon, R. C., Pisat, N., Johnson, S. L. \& Dougherty, J. D. Development of translating ribosome affinity purification for zebrafish: Zebrafish Translating Ribosome Purification. Genesis 51, 187-192 (2013).

27. Thomas, A. et al. A versatile method for cell-specific profiling of translated mRNAs in drosophila. PLoS ONE 7, e40276 (2012).

28. Carayon, A. et al. Intrinsic control of muscle attachment sites matching. Life 9, e57547 (2020).

29. Clemen, C. S., Herrmann, H., Strelkov, S. V. \& Schröder, R. Desminopathies: pathology and mechanisms. Acta Neuropathol (Berl) 125, 47-75 (2013).

30. Naddaf, E., Barohn, R. J. \& Dimachkie, M. M. Inclusion body myositis: update on pathogenesis and treatment. Neurotherapeutics 15, 995-1005 (2018).

31. Page, L. J. et al. Secretion of amyloidogenic gelsolin progressively compromises protein homeostasis leading to the intracellular aggregation of proteins. Proc. Natl. Acad. Sci. 106, 11125-11130 (2009).

32. Gratz, S. J. et al. Highly specific and efficient CRISPR/Cas9-catalyzed homology-directed repair in drosophila. Genetics 196, 961-971 (2014).

33. Port, F., Chen, H.-M., Lee, T. \& Bullock, S. L. Optimized CRISPR/Cas tools for efficient germline and somatic genome engineering in Drosophila. Proc. Natl. Acad. Sci. 111, E2967-E2976 (2014).

34. Isaacs, E. R., Bradley, W. G. \& Henderson, G. Longitudinal fibre splitting in muscular dystrophy: a serial cinematographic study. Neurol. Neurosurg. Psychiatry. 36, 813-819 (1973).

35. Gutiérrez, J. M. et al. Skeletal muscle degeneration and regeneration after injection of bothropstoxin-II, a phospholipase $A_{2}$ isolated from the venom of the snake Bothrops jararacussu. Exp. Mol. Pathol. 55, 217-229 (1991).

36. Chan, S. \& Head, S. I. The role of branched fibres in the pathogenesis of Duchenne muscular dystrophy. Exp. Physiol. 96, 564-571 (2011).

37. Pichavant, C. \& Pavlath, G. K. Incidence and severity of myofiber branching with regeneration and aging. Skelet Muscle 4, 9 (2014).

\section{Acknowledgements}

This work was supported by AFM-Téléthon (MyoNeurAlp Strategic Program), Agence Nationale de la Recherche (Tefor Infrastructure Grant), ANR JC (Cardiac-SPE), Fondation pour la Recherche Médicale (Equipe FRM Award) and the iSITE CAP2025 Grant. We thank the staff of Genecore EMBL Heidelberg for their technical assistance.

\section{Author contributions}

B.B., Y.R., T.J., G.L., C.D., J.P.D. performed experiments, G.J. and K.J. designed experiments, analysed data and wrote the manuscript.

\section{Competing interests}

The authors declare no competing interests.

\section{Additional information}

Supplementary Information The online version contains supplementary material available at https://doi.org/ 10.1038/s41598-021-92506-3.

Correspondence and requests for materials should be addressed to G.J. or K.J.

Reprints and permissions information is available at www.nature.com/reprints.

Publisher's note Springer Nature remains neutral with regard to jurisdictional claims in published maps and institutional affiliations.

(c) (i) Open Access This article is licensed under a Creative Commons Attribution 4.0 International License, which permits use, sharing, adaptation, distribution and reproduction in any medium or format, as long as you give appropriate credit to the original author(s) and the source, provide a link to the Creative Commons licence, and indicate if changes were made. The images or other third party material in this article are included in the article's Creative Commons licence, unless indicated otherwise in a credit line to the material. If material is not included in the article's Creative Commons licence and your intended use is not permitted by statutory regulation or exceeds the permitted use, you will need to obtain permission directly from the copyright holder. To view a copy of this licence, visit http://creativecommons.org/licenses/by/4.0/.

(C) The Author(s) 2021 